\title{
Electron microscopy of chlorphentermine lung
}

\author{
PAUL SMITH, DONALD HEATH, and P. S. HASLETON \\ The Department of Pathology, University of Liverpool
}

\begin{abstract}
Smith, P., Heath, D., and Hasleton, P. S. (1973). Thorax, 28, 559-566. Electron microscopy of chlorphentermine lung. Electron microscopy of lung tissue from rats treated with chlorphentermine shows an intense hyperplasia in the alveolar walls of granular pneumocytes which remain attached and do not migrate into the alveolar spaces. Numerous 'foam cells' are found free in the alveoli and are histiocytes which have ingested osmiophilic lamellar bodies. Similar laminated inclusions are found in granular pneumocytes, membranous pneumocytes, bronchiolar Clara cells, pulmonary macrophages, and endothelial cells of pulmonary capillaries. The intra-alveolar debris is rich in osmiophilic lamellar bodies with associated phospholipid lattices. The appearances are those of a toxic alveolitis. Further studies of chronic toxicity are suggested to ascertain if this acute lesion progresses to fibrosing alveolitis.
\end{abstract}

In the preceding paper we showed that administration of chlorphentermine for 50 days to rats does not give rise to hypertensive pulmonary vascular disease but instead leads to pronounced changes in the lung parenchyma (Heath, Smith, and Hasleton, 1973). These consist of a cellular hyperplasia of the alveolar walls and an accumulation of 'foam cells' in the alveolar spaces. In the present study we identified these cells in the alveolar walls and spaces by electron microscopy and studied the ultrastructure of the associated intra-alveolar debris.

\section{METHODS}

A piece of lung tissue for electron microscopy was taken from the lateral third of the superior lobe of the right lung in each rat. This was placed on a piece of card in a pool of glutaraldehyde and cut into small fragments measuring approximately $1 \mathrm{~mm}$ across. These were transferred to bottles containing ice-cold buffered glutaraldehyde and allowed to fix for 24 hours. The blocks of tissue were washed in isotonic sucrose solution and post-fixed in $1 \%$ buffered osmium tetroxide for 1 hour before being embedded in Araldite. Thin sections $(1 \mu \mathrm{m})$ were cut and stained with toluidine blue for the selection of suitable areas for electron microscopic examination. The blocks were then trimmed and fine sections were cut with an LKB Ultrotome III ultramicrotome to obtain silver-coloured sections. The sections were floated onto copper grids, stained with uranyl acetate and lead citrate, and examined with an AEI EM6B electron microscope using an acceleration voltage of $60 \mathrm{kV}$.

\section{RESULTS}

There was an intense hyperplasia of cells attached to the alveolar walls. These had the ultrastructural characteristics of granular (type II) pneumocytes which have been described elsewhere (Brewer, Heath, and Asquith, 1969). In particular, the cells contained many lamellar bodies (Fig. 1). These granular pneumocytes were shaped like the segment of a circle, the chord of this segment being represented by the straight surface of the cell which was attached to the alveolar wall. The curved surfaces of the cells projecting into the alveolar space were covered by short straight microvilli (Fig. 1). Such ultrastructural features were found only in cells still attached to the alveolar wall.

Many large cells were lying free in the alveolar spaces and they contained large and numerous osmiophilic lamellar inclusions (Fig. 2). At first sight they appeared to be granular pneumocytes but on closer examination they included lysosomal dark bodies, many of which surrounded, or were in close association with, lamellar material. These cells appeared to be macrophages which had ingested material derived from the true lamellar bodies of granular pneumocytes (Figs 3 and 4). In this paper we shall restrict the term 'lamellar body' to the osmiophilic laminated bodies found only in a recognizable granular pneumocyte. We shall refer to laminated material of similar structure in other cells as osmiophilic lamellar 

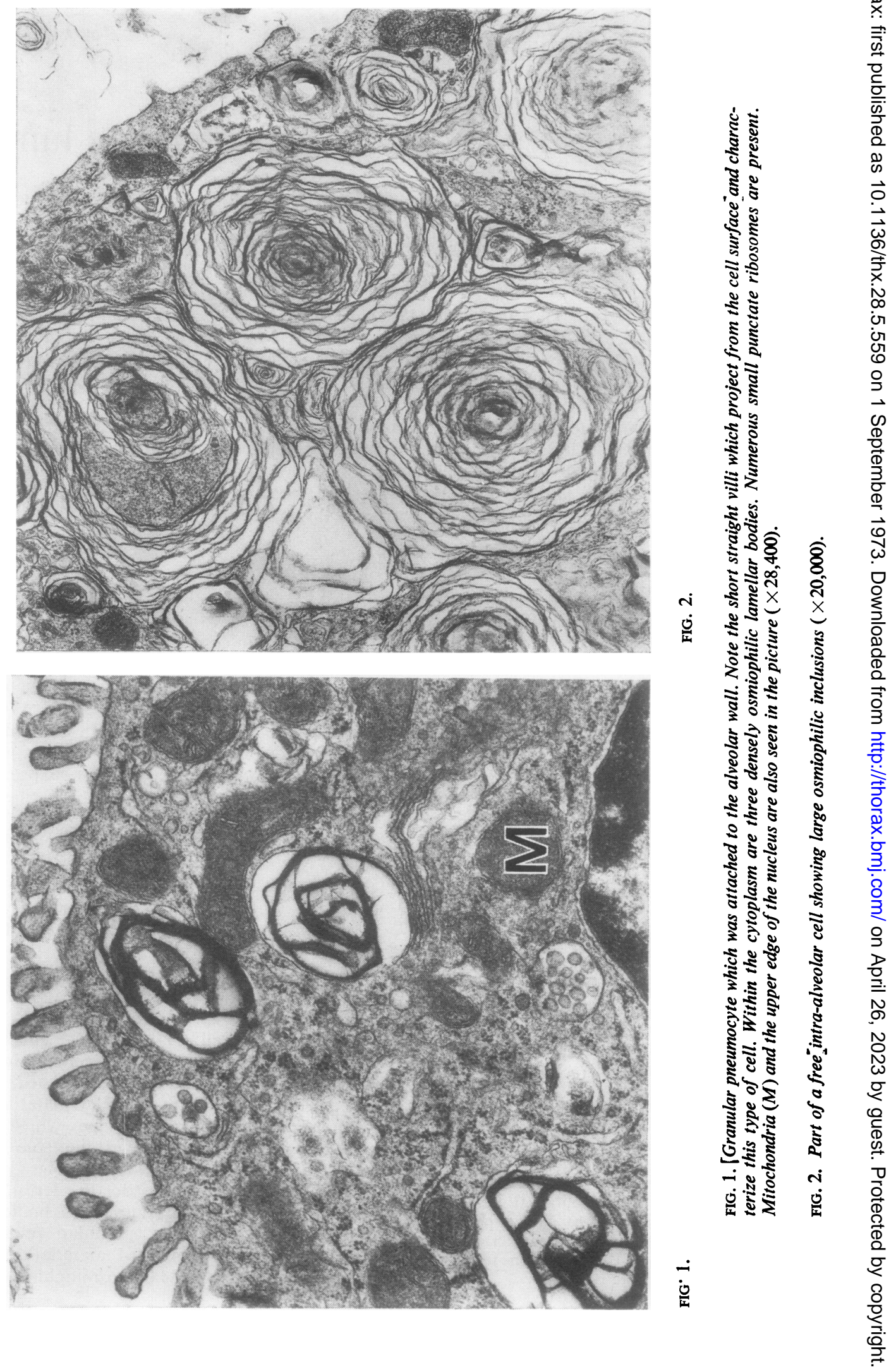

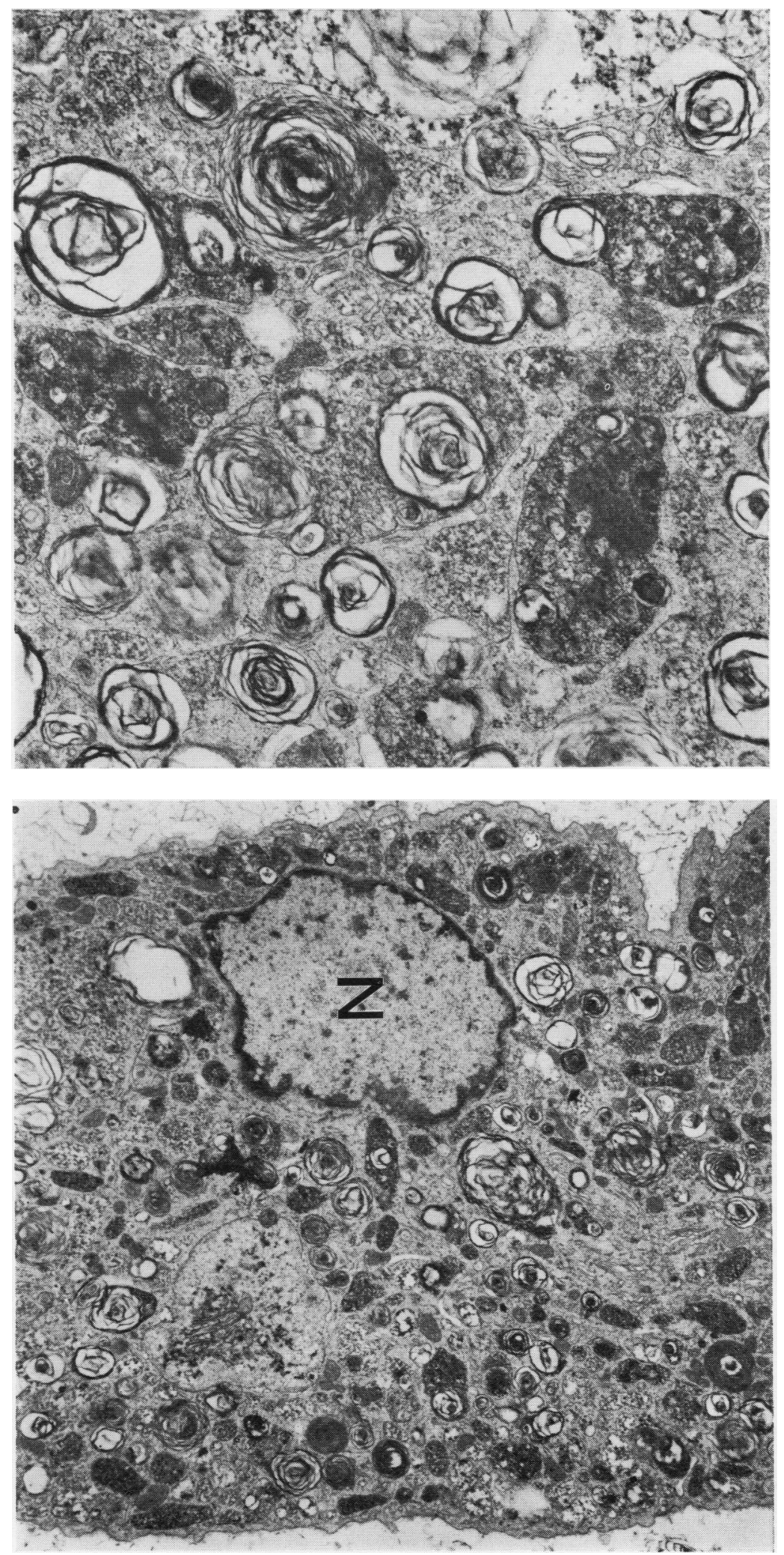

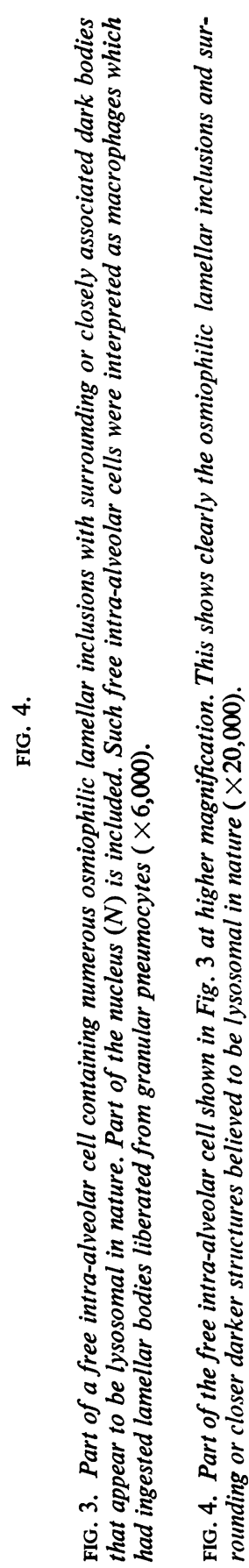



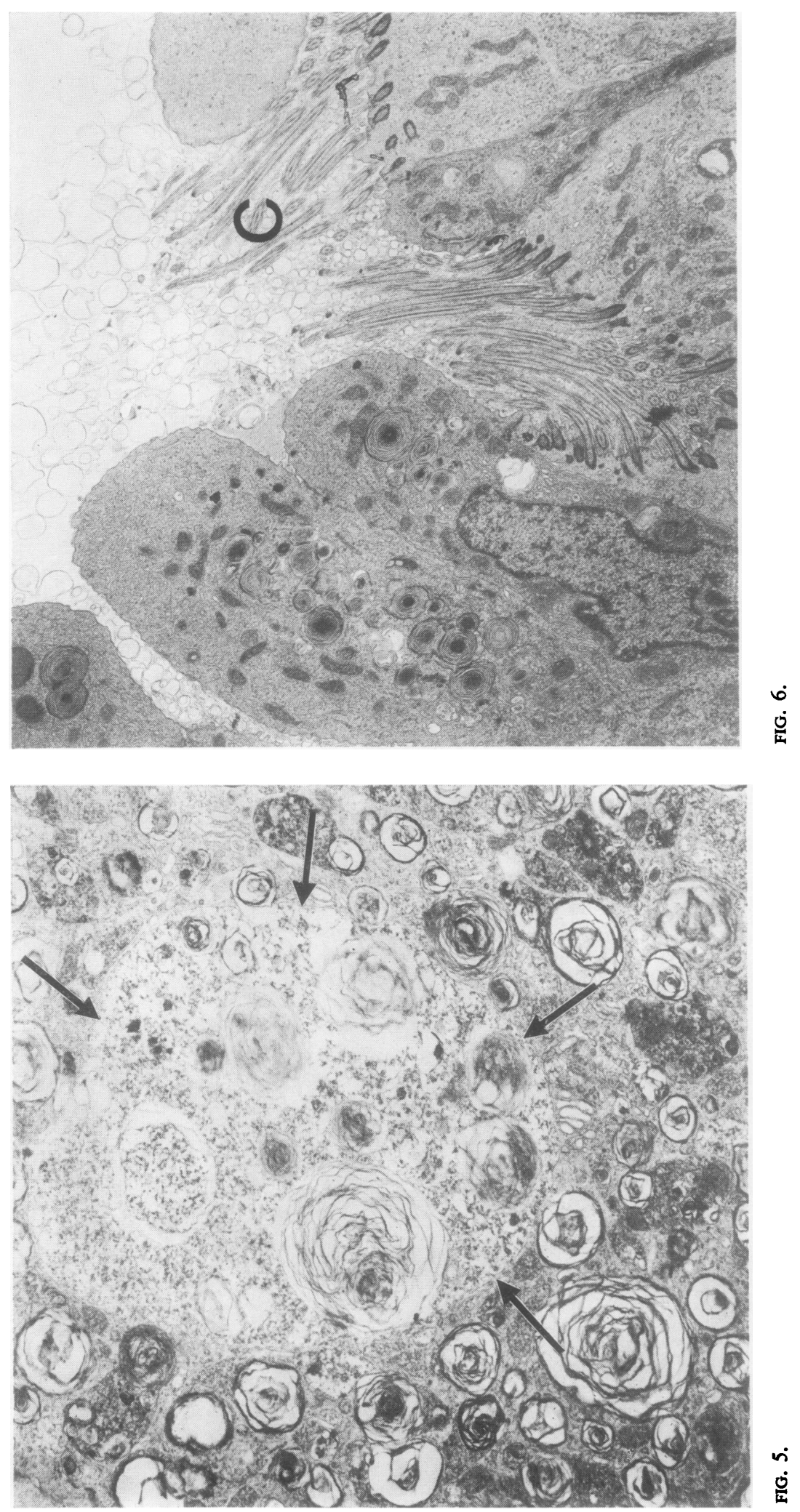

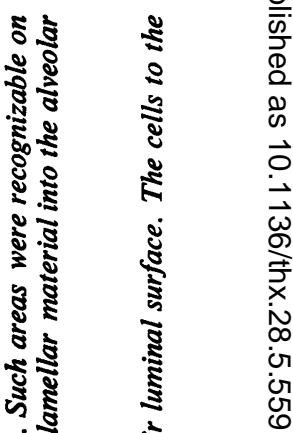

ริธ 


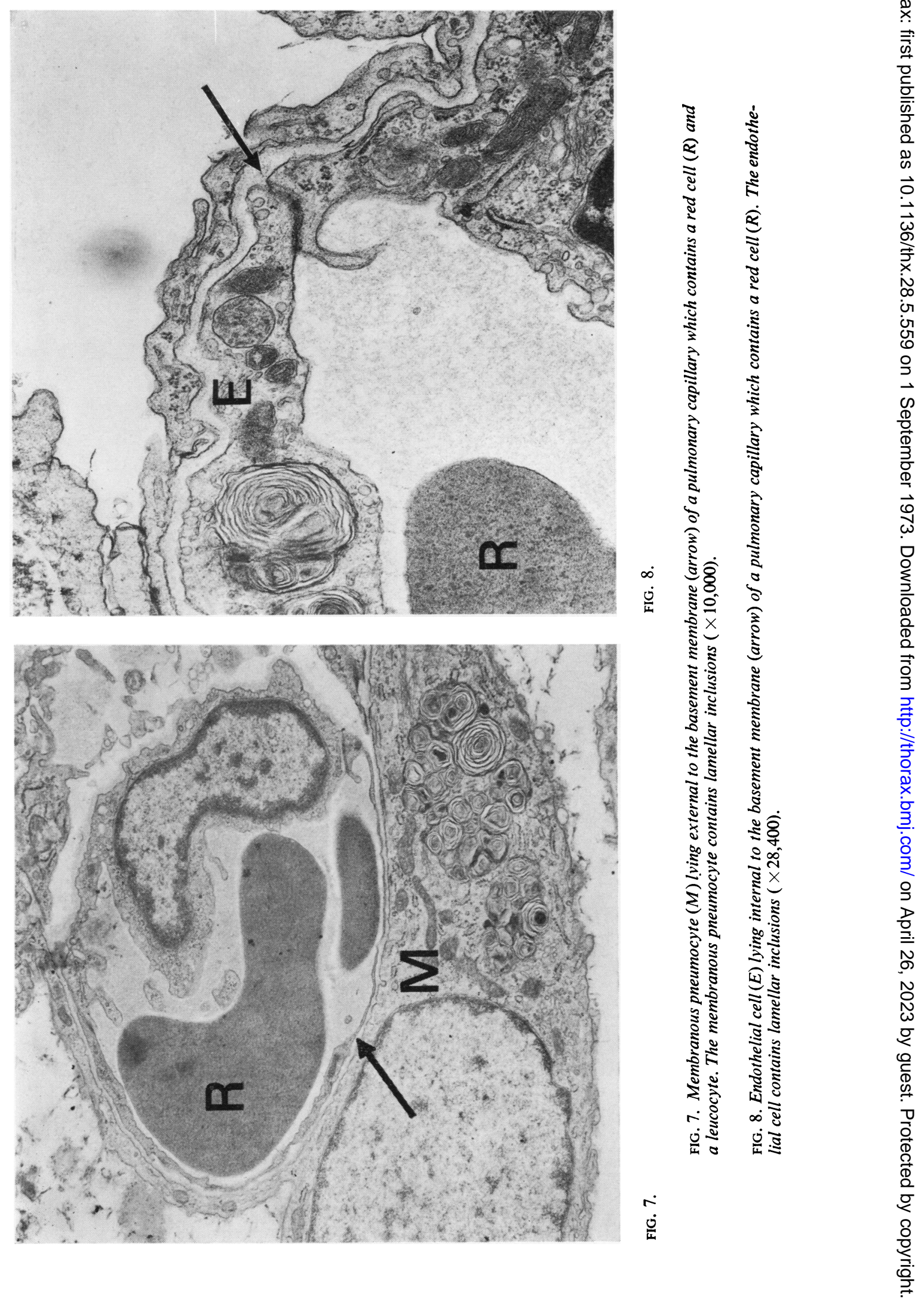



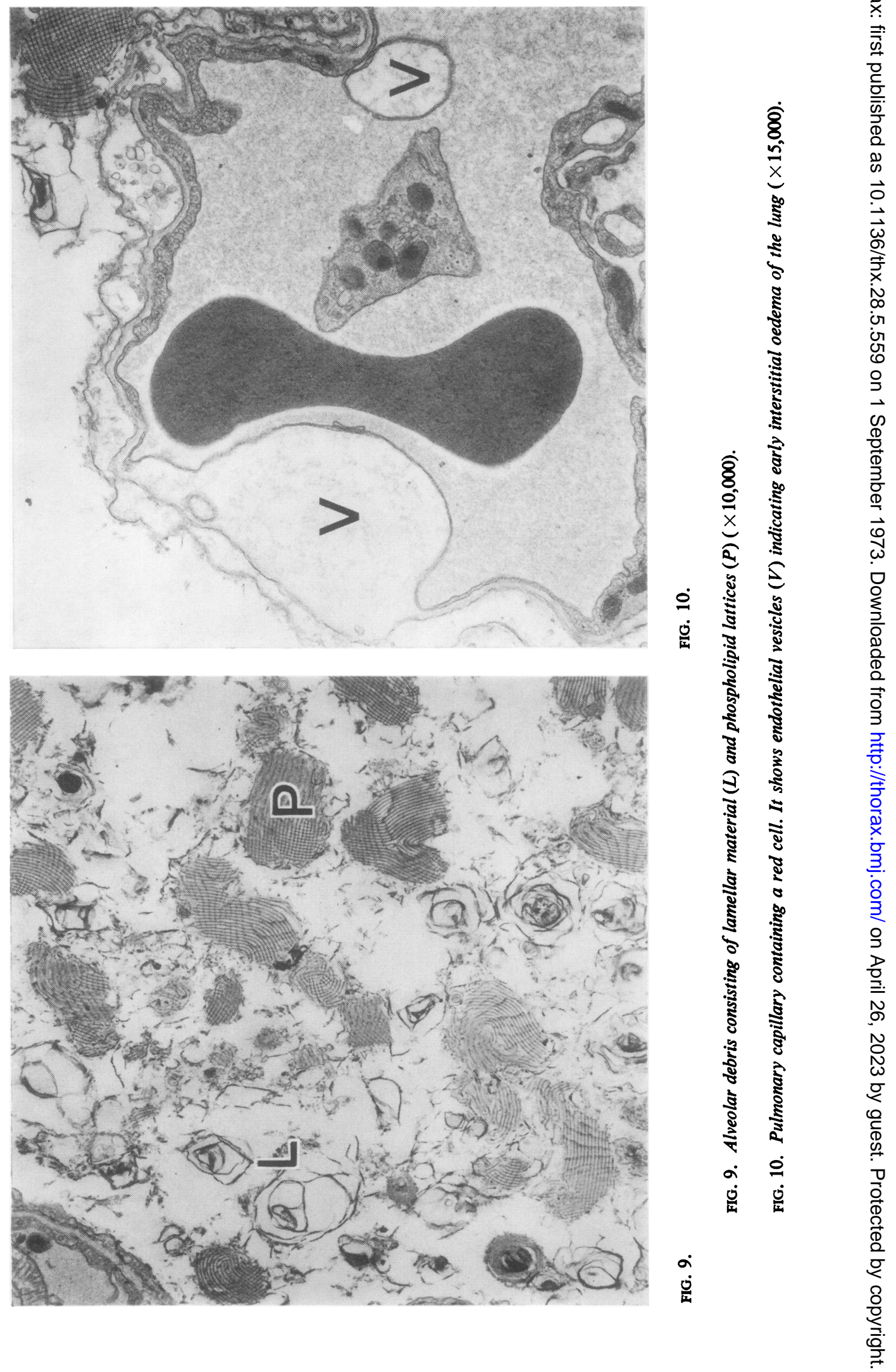
inclusions. Virtually all cells lying free in alveolar spaces were macrophages of this type. All the cells that could be identified positively as granular pneumocytes were attached to alveolar walls. Many of the intra-alveolar cells showed central pale areas in which the cytoplasm and its contained organelles appeared to be in the process of disintegration (Fig. 5). Such areas of pallor clearly corresponded to the pale-staining areas in intraalveolar cells that we had previously observed on light microscopy (Heath et al., 1973).

Lamellar material was not confined to granular pneumocytes and intra-alveolar macrophages. Osmiophilic lamellar inclusions were also found abundantly within the bronchioles in Clara cells which are devoid of cilia and quite distinct in structure from the respiratory epithelial cells (Fig. 6). They were also seen within the cytoplasm of membranous type I pneumocytes which may have engulfed this material from the debris lying free in alveolar spaces (Fig. 7). Alternatively, the presence of osmiophilic lamellar bodies in these cells may be an expression of cellular degeneration. The membranous pneumocyte could be identified by its flattened, attenuated form and its position between the fused basement membranes of the alveolar wall and the cavity of the alveolus (Fig. 7). Endothelial cells, lining pulmonary capillaries and lying within the fused basement membranes of the alveolar wall, also contained lamellar inclusions (Fig. 8). Hence osmiophilic lamellar inclusions were found in no fewer than five classes of cell in the lung, namely the granular pneumocyte, the membranous pneumocyte, the Clara cell, the intra-alveolar macrophage, and the endothelial cell. It is not surprising that lamellar material of this type was so widespread since the alveolar spaces contained large numbers of disintegrating intra-alveolar cells and much debris resulting from this disintegration. As a result the alveoli were packed with lamellar material and lattices of phospholipid (Fig. 9). The combination of substances corresponds to the apparently amorphous debris staining a cherry-pink colour with the periodic acid Schiff stain that was found on light microscopy. The intra-alveolar cells staining a magenta colour with this stain correspond to intra-alveolar macrophages.

Electron microscopy also revealed the early stages of interstitial pulmonary oedema with the production of blebs and endothelial vesicles which protruded into the lumina of the pulmonary capillaries (Fig. 10). There was no evidence of early interstitial pulmonary fibrosis.

\section{DISCUSSION}

Our study shows that in the lungs of rats receiving chlorphentermine there is an intense hyperplasia of granular pneumocytes in the alveolar walls. Their cytoplasm becomes filled with lamellar bodies and it seems likely that many of these are liberated into the alveoli. Significantly we found none of these granular pneumocytes lying free in the alveolar spaces; they always remained attached to the alveolar walls. This suggests that the 'foam cells' found so profusely in the alveolar spaces on light microscopy (Heath et al., 1973) are not granular pneumocytes but another variety of cell.

The ultrastructural features of the free intraalveolar cells, which gave a magenta colour with the PAS stain on light microscopy, suggest that they are macrophages. They present difficulty in identification because they ingest so much of the intra-alveolar debris rich in osmiophilic lamellar material and phospholipid that they come to resemble granular pneumocytes. Their true identity is revealed by the presence of lysosomal bodies. Groups of these pulmonary histiocytes congregate together in alveoli and then undergo disintegration to give rise to the particulate intraalveolar debris which on light microscopy stained a cherry-pink colour with the PAS stain. As we have seen, this debris is packed with osmiophilic lamellar material and phospholipid lattices.

Chlorphentermine certainly produces a remarkable pathological picture in the lungs of rats with osmiophilic lamellar inclusions abounding not only free in the alveolar spaces but in no fewer than five classes of cell, namely the granular (type II) pneumocyte, the membranous (type I) pneumocyte, the bronchiolar Clara cell, the intraalveolar macrophage, and the endothelial cells of pulmonary capillaries. Nevertheless, this remarkable pulmonary histiocytosis and profusion of lamellar material is in no sense specific for chlorphentermine administration. Virtually identical changes were noted by Vijeyaratnam and Corrin (1972a) in the lungs of rats receiving oral iprindole. This imino dibenzyl derivative is used clinically as an antidepressant drug and when given to rats gives rise to an accumulation of cells in the alveolar spaces similar in appearance to those which we have seen in desquamative interstitial pneumonia in man (Brewer et al., 1969). Vijeyaratnam and Corrin (1972a) found that the free intra-alveolar cells showed high acid phosphatase and $\beta$ glucuronidase activity, suggesting that they were macrophages. This was confirmed by electron microscopy and by the avid ingestion of 
thorium particles introduced via the trachea. These authors were convinced that the iprindole lesion represents a pulmonary histiocytosis rather than an alveolar epithelial desquamation and this mirrors our own opinion of the chlorphentermine lesion.

The origin of the free pulmonary histiocytes was demonstrated by Vijeyaratnam and Corrin (1972b), who studied mitotic counts and the ultrastructure of the lungs of iprindole-treated rats. They found that the pulmonary histiocytosis was accompanied by proliferation of cells in the interstitial tissues of the lung which they regarded as the immediate precurors of the alveolar macrophages. They point out that such findings are not incompatible with cells derived from extrapulmonary tissues, such as bone marrow, undergoing further division and maturation within the interstitial tissues of the lung before entering the air-spaces as alveolar macrophages.

The interstitial oedema that we have found in the lungs of rats treated by chlorphentermine (Fig. 10) was also noted by Vijeyaratnam and Corrin (1973) in their studies on iprindole. They found a leakage of thorium particles which had been injected intravenously and they noted-as we have in the case of chlorphenterminedegenerative changes in the endothelial cells of pulmonary capillaries. This suggested to them that the pulmonary oedema was due to increased capillary permeability rather than to raised intravascular pressure. Just as in the case of chlorphentermine Vijeyaratnam and Corrin found lamellar osmiophilic inclusions in all five classes of pulmonary cell that we list above.

The present study reveals only the ultrastructural details of the acute stage of the pulmonary disease induced by the administration of chlorphentermine; it does not demonstrate the course and nature of the chronic lesions that must follow those which we have described here. The profuse hyperplasia of granular pneumocytes following the administration of a drug is somewhat reminiscent of busulphan lung, a disease characterized by the subsequent development of fibrosing alveolitis (Littler, Kay, Hasleton, and Heath, 1969). In view of this we think it desirable to carry out chronic studies with chlorphenter- mine to see if the acute lesions described here will progress to fibrosing alveolitis. In other words, $\overparen{\varnothing}$ it is important to ascertain if the predominanto action of the drug is on the lung parenchyma. In $_{\triangle}^{\text {\& }}$ view of the continuing controversy surrounding. the suspected relation between anorexigens and $\overrightarrow{\vec{\omega}}$ pulmonary hypertension it seems equally im- $\stackrel{\omega}{\circ}$ portant to exclude the possibility that chronic $\vec{x}$ administration of the drug may lead to involve-i ment of the pulmonary vasculature with $\mathrm{O}$ hypertensive pulmonary vascular disease.

Vijeyaratnam and Corrin (1972a) note that pul- $-\mathcal{C}_{0}$ monary histiocytosis is common in rats ando considered that the absence of this cellular exudation in other species justified the use of $c s$ iprindole in clinical trials. On the other hand, $\frac{\mathbb{D}}{0}$ we feel that chlorphentermine is only one of $\frac{\mathbb{\Phi}}{3}$ many anorexigens available to the medical profession and doubt the wisdom of prescribing a drug that can lead to such dramatic changes in $\overrightarrow{0}$ the lung parenchyma of a laboratory animal. It $\omega$ should be noted here that similar changes are not produced by the anorexigen aminorex fumarate at present suspected of causing pulmonary hypertension. In our previous studies on this drug (Kay, Smith and Heath, 1971) we have not $\AA$ found the remarkable proliferation of pulmonary histiocytes such as is produced by chlorphen- $\frac{0}{3}$ termine.

\section{REFERENCES}

Brewer, D. B., Heath, D., and Asquith, P. (1969). Electron microscopy of desquamative interstitial pneumonia. Journal of Pathology, 97, 317.

Heath, D., Smith, P., and Hasleton, P. S. (1973). Effects of chlorphentermine on the rat lung. Thorax, 28, 551.

Kay, J. M., Smith, P., and Heath, D. (1971). Aminorex and the pulmonary circulation. Thorax, 26, 262.

Littler, W. A., Kay, J. M., Hasleton, P. S., and Heath, D. (1969). Busulphan lung. Thorax, 24, 639.

Vijeyaratnam, G. S., and Corrin, B. (1972a). Pulmonary histiocytosis simulating desquamative interstitial pneu- $N$ monia in rats receiving oral iprindole. Journal of Pathology, 108, 105.

and $-(1972 b)$. Origin of the pulmonary alveolar $N$ macrophage studied in the iprindole-treated rat. Journal of Pathology, 108, 115.

- and $-(1973)$. Fine structural alterations in the lungs of iprindole-treated rats. Journal of Pathology (In press). 